\title{
Design and Optimization of a Microfiber Coupler for Biosensing
}

\author{
Rand Ismaeel, ${ }^{1}$ * Timothy Lee, ${ }^{1}$ Marcelo A. Gouveia, ${ }^{1}$ Ming Ding, ${ }^{1}$ Tracy Melvin, ${ }^{1}$ and Gilberto Brambilla ${ }^{1}$ \\ ${ }^{I}$ Optoelectronics Research Centre, University of Southampton, SO17 1BJ, United Kingdom. \\ *Corresponding author: rmni1g10@orc.soton.ac.uk
}

\begin{abstract}
We design and optimize a simple, low-cost and compact biosensor based upon a microfiber coupler, with a predicted sensitivity and detection limit up to $\mathrm{S} \sim 10^{4} \mathrm{~nm} / \mathrm{RIU}$ and DL $\sim 10^{-6}$ RIU respectively.

OCIS codes: (280.0280) Remote sensing and sensors; (280.1415) Biological sensing and sensors
\end{abstract}

Simple, direct bioanalytical approaches with excellent quantifiable limits of detection are becoming ever more important for medical diagnosis and management of patient care. Although the majority of the biosensors developed so far are based upon assays requiring a labeled molecule (i.e. fluorophore) [1-3], a variety of label-free biosensors have been developed; these include, amongst others, surface plasmon resonance sensors [4] and photonic crystal sensors [5]. Yet, most of these sensors suffer from being costly and difficult to fabricate. Therefore, the development of cost effective and simple label-free biosensors is still necessary. Indeed, recently others have applied optical fibre technology as a promising new approach for DNA detection [6-8, thus in this vein here we investigate and develop compact silica microfiber couplers for DNA refractometric sensing with high limits of detection.

In recent years, microfibers $[9,10]$ have gained significant interest in variety of fields, especially in sensing applications [11-12] where the large evanescent field observed for microfibers allows the detection of changes in the surrounding medium. Furthermore, both the sensitivity and detection limit of the microfiber sensor could be tailored to the concentration of the DNA attached to the coupler waist by altering the coupler geometry. DNA binding introduces a refractive index change of the range of $\Delta \mathrm{n} \sim 10^{-5}-10^{-2}$ for DNA concentrations in the range $0.5-2 \mu \mathrm{g} / \mathrm{mL}$ $[8,13,14]$ which would then induce a change in the transmission spectrum and thus in the power splitting ratio at a fixed wavelength.

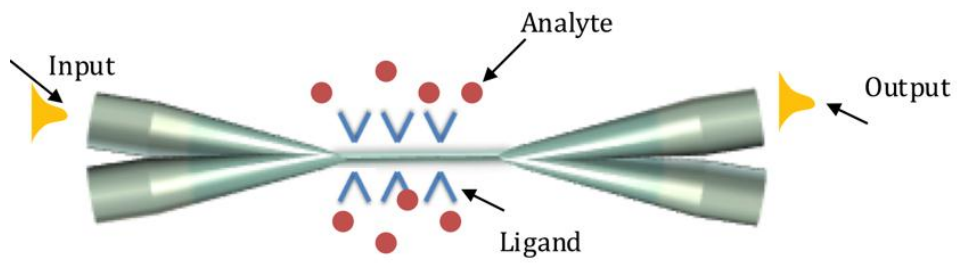

Figure 1: Schematic of the coupler sensor.

The change in the index can be deduced by measuring the wavelength shift of the coupler's transmission dip. In order to investigate the coupler sensitivity at different diameters, the following equations $[15,16]$ were used to explore the behavior of couplers with different diameters:

$$
P=\frac{1}{2}\left[1+\cos \left(C_{x}+C_{y}\right) L \cos \left(C_{x}-C_{y}\right) L\right]
$$

where $P$ represent the power at one of the output ports, $L$ the length of the fused region and $C_{x, y}$ are the coupling strengths for the $\mathrm{x}$ and $\mathrm{y}$ polarized modes:

$$
\begin{gathered}
C_{x}+C_{y}=\frac{3 \pi \lambda}{32 n_{2} a^{2}}\left[\frac{1}{\left(1+\frac{1}{V}\right)^{2}}+\frac{1}{\left(1+\frac{n_{2}^{2}}{n_{1}^{2}} \cdot \frac{1}{V}\right)^{2}}\right] \\
C_{x}-C_{y}=\frac{3 \pi \lambda}{16 n_{2} a^{2}} \cdot \frac{1}{V}\left(1-\frac{n_{2}^{2}}{n_{1}^{2}}\right)
\end{gathered}
$$

where $a$ is the coupler radius, and $n_{1}$ and $n_{2}$ are the core and cladding indices respectively. Solving the equations above showed that the sensitivity of the coupler increases with the increasing wavelength and decreasing diameter of 
the coupler waist. By optimizing these parameters, the sensitivity can reach up to $S \sim 10^{4} \mathrm{~nm} / \mathrm{RIU}$, where couplers with smaller diameters (Figure 2(a)) have even higher sensitivities. However, the dynamic range of the coupler, determined by the distance between the maxima and the minima of two consecutive transmission dips and shown in Figure 2(b), is reduced at smaller diameters, thus it limits the range of DNA concentrations that can be detected. Therefore, it is necessary to select the right diameter for the given DNA concentration, although even with high concentrations (i.e more than $1 \mu \mathrm{g} / \mathrm{mL}$ ) the wavelength shift does not exceed $50 \mathrm{~nm}$ and so a coupler diameter of, for example, $4 \mu \mathrm{m}$ still offers good dynamic range as well as high sensitivity.
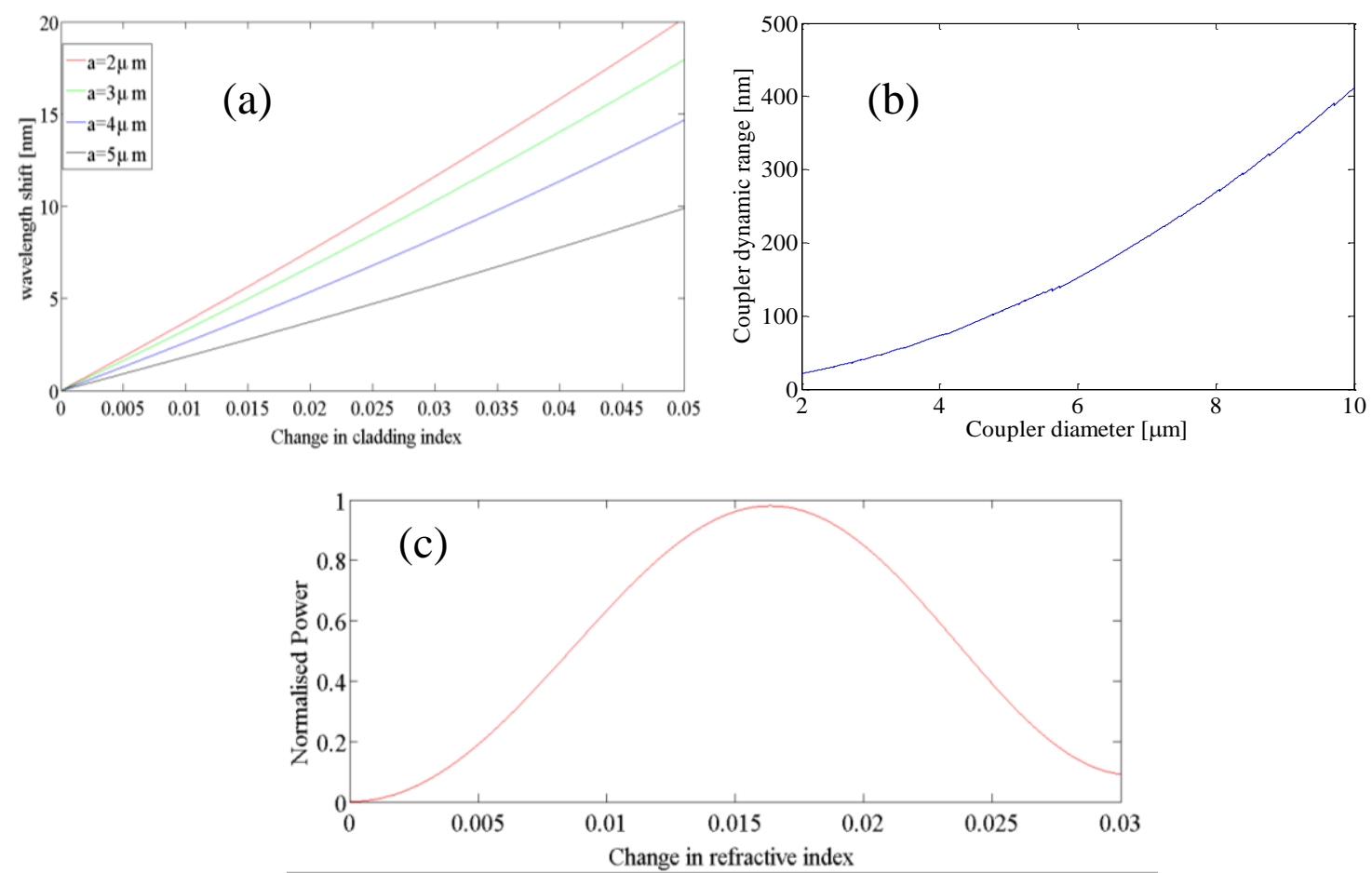

Figure 2. (a) Sensitivity of the coupler to the refractive index change at different coupler diameters. (b) Coupler dynamic range at different diameters (c) Change in output power with respect to the change in the surrounding index at fixed wavelength.

It is also possible to detect the change in index induced by the deposited DNA by monitoring the change in the output power at single wavelength as shown in Figure 2(c). This method provides an enhanced detection limit up to DL $\sim 0^{-6}$ RIU, However, the output power is uniquely dependent on the refractive index change of the surrounding but only for DNA concentration in the range of $(0-0.017 \mu \mathrm{g} / \mathrm{mL})$. The calculated change in the power as a result of the index change (Fig 2. (c)), matches that experimentally obtained in previous publication [14].

The fabrication of the device included heating and pulling two single mode fibers together under uniform heat as well as controlled pulling speed until the two fibers were fused together. The process was then stopped when the desired coupler transmission was observed on an optical spectrum analyzer connected to the coupler output. The coupler diameter was chosen to be $5 \mu \mathrm{m}$ to allow maximum dynamic range for high DNA concentration, and the recorded spectra are shown in Figure 3.

The coupler was cleaned and then functionalized using a $1 \%$ solution of distilled trimethoxysilylpropyldiethylenetriamine in $1 \mathrm{mM}$ acetic acid in deionized (dI) $\mathrm{H}_{2} \mathrm{O}$ for $20 \mathrm{~min}$ at room temperature, rinsed three times with dI water and dried under $\mathrm{N}_{2}$. The surface functionalized coupler was then modified with the heterobifunctional crosslinker, $\mathrm{N}$-( $\gamma$-maleimidobutryloxy) succinimide ester (GMBS), and prepared as $1 \mathrm{mM}$ solutions. Crosslinkers were dissolved in $100 \mu \mathrm{L}$ DMSO and diluted to $30 \mathrm{ml}$ in 80:20 MeOH:DMSO. The silyl functionalised coupler was immersed in the GMBS crosslinker solution for 2 hours at room temperature, and then rinsed with the solvent used for dilution and dried under $\mathrm{N}_{2}$. The transmission of the coupler at this point represents a reference for the change that the DNA deposition would induce for the output of the coupler. The following steps include DNA synthesis and purification as well as treatment of crosslinker-modified substrates with thiolated DNA oligomers in a method similar to that mentioned in [17]. 


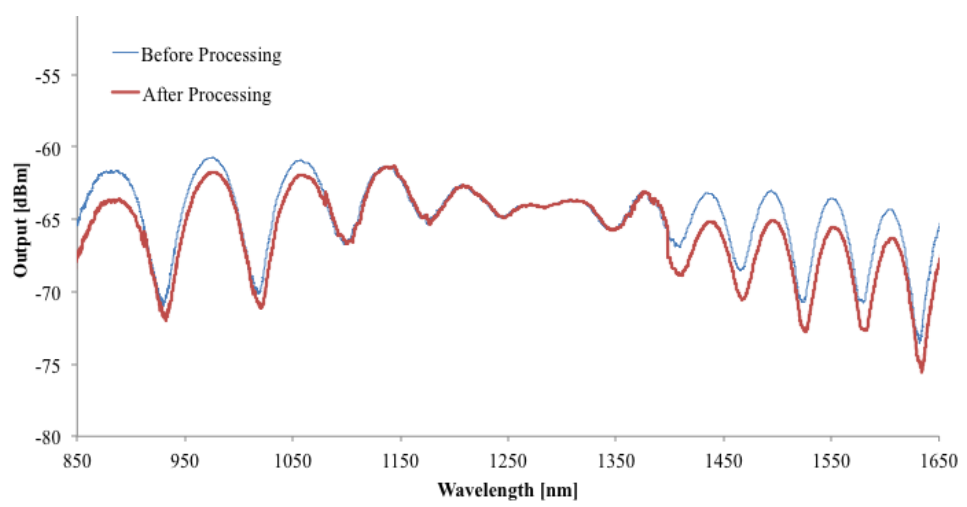

Figure 3: The experimentally observed power output spectra of the coupler before and after covalent attachment of the GMBS crosslinker deposition.

For attachment of DNA of the required sequence, a 5'-end labeled thiol-DNA oligonucleotide in deaerated $10 \mathrm{mM}$ HEPES, $5 \mathrm{mM}$ EDTA buffer, pH 6.6 is used. Concentrations of the DNA solution were of the order of $1 \mu \mathrm{g} / \mathrm{mL}$. The GMBS-functionalized coupler was immersed in the thiolated-DNA solution for 2 hours at room temperature, then rinsed and dried under $\mathrm{N} 2$. The spectrum of the coupler was recorded before and after this stage.

In summary, simulations showed that an optical fiber coupler optimized for the purpose of DNA sensing can have sensitivity and detection limit of $\mathrm{S} \sim 10^{4} \mathrm{~nm} / \mathrm{RIU}$ and $\mathrm{DL} \sim 10^{-6}$ RIU respectively. The fabrication and DNAfunctionalization of the simple microfiber biosensor is demonstrated.

\section{References}

[1] C. R. Taitt, G. P. Anderson, F. S. Ligler, "Evanescent wave fluorescence biosensors," Biosensors and Bioelectronics 20(12), pp. 2470-2487 (2005).

[2] Y. Chang, R. Chen, Y. Lee, S. Chao, L. Su, Y. Li, C. Chou, "Localized surface plasmon coupled fluorescence fiber-optic biosensor for alphafetoprotein detection in human serum," Biosensors and Bioelectronics 24(6), pp. 1610-1614 (2009).

[3] G. P. Anderson, J. P. Golden, F. S. Ligler, "An evanescent wave biosensor. I. Fluorescent signal acquisition from step-etched fiber optic probes,” Biomedical Engineering, IEEE 41(6), pp. 578-584 (1994).

[4] J. Homola, "Optical fiber sensor based on surface plasmon excitation," Sensors and Actuators B: Chemical 29, pp. 401-405 (1995)

[5] N. Skivesen, A. Têtu, M. Kristensen, J. Kjems, L. Frandsen, P. Borel, "Photonic-crystal waveguide biosensor," Opt. Express 15, pp. 31693176 (2007).

[6] M. J. Yin, C. Wu, Y. L. Shao, W. K. Chan, A. P. Zhang, C. Lu, H. Y. Tam, "Label-free, disposable fiber-optic biosensors for DNA hybridization detection," Analyst 138(7), pp. 1988-1994 (2013).

[7] J. Ott, M. Heuck, C. Agger, P. Rasmussen, O. Bang, "Label-free and selective nonlinear fiber-optical biosensing," Opt. Express 16, pp. 20834-20847 (2008).

[8] X. Fan, I. M. White, A. I. Shopova, H. Zhu, J. D. Suter, "Sensitive optical biosensors for unlabeled targets: A review," Analytica Chimica Acta 620, pp. 8-26 (2008).

[9] G. Brambilla, “Optical fiber nanowires and microwires: a review,” J. Opt. 12(4), 043001 (2010).

[10] L. M. Tong, R. R. Gattass, J. B. Ashcom, S. L. He, J. Y. Lou, M. Y. Shen, I. Maxwell, E. Mazur, "Subwavelength-diameter silica wires for low-loss optical wave guiding," Nature 426, pp. 816-819 (2003).

[11] F. Xu, G. Brambilla, "Demonstration of a refractometric sensor based on optical microfiber coil resonator," Appl. Phys. Lett. 92, 101126 (2008).

[12] M. Belal, Z. Song, Y. Jung, G. Brambilla, T. P. Newson, "Optical fiber microwire current sensor," Opt. Lett. 35, pp. 3045-3047 (2010).

[13] B. Luff, R. Harris, J. Wilkinson, R. Wilson, D. Schiffrin, "Integrated-optical directional coupler biosensor," Opt. Lett. 21, pp. 618-620 (1996).

[14] H. Tazawa, T. Kanie, "Fiber-optic coupler biosensor," in IEEE Sensors, Atlanta 28-31 Oct. 2007, pp.604-607.

[15] F. P. Payne, C. D. Hussey, M. S. Yataki, "Polarisation analysis of strongly fused and weakly fused tapered couplers," Electron. Lett. 21(13), pp. 561-563 (1985).

[16] M. Ding, P. Wang, G. Brambilla, “A microfiber coupler tip thermometer,” Opt. Express 20, pp. 5402-5408 (2012).

[17] L. A. Chrisey, G. U. Lee, .C. E. O'Ferrall, "Covalent Attachment of Synthetic DNA to Self-Assembled Monolayer Films," Nucleic Acids Research 24, pp. 3031-3039 (2012). 